\title{
SOME SEMI-ClASSICAL AND LAGUERRE-HAHN FORMS DEFINED BY PSEUDO-FUNCTIONS
}

\section{J. Alaya and P. Maroni}

\begin{abstract}
We deal with some examples of semi-classical forms defined by Hadamard finite parts. We construct corresponding orthogonal sequences and their respective structure relations. Inverse and associated forms also are considered. Representation of these forms are given. In general, they are Laguerre-Hahn forms.
\end{abstract}

\section{Introduction}

In a recent paper [16], the second author has studied the generalized weights $P f \frac{V(x)}{x^{2}}$ defined by

$$
\begin{aligned}
\left\langle P f \frac{V(x)}{x^{2}}, f\right\rangle & =P f \int_{-\infty}^{\infty} \frac{V(x)}{x^{2}} f(x) d x \\
& =\lim _{\varepsilon \rightarrow+0}\left(\int_{-\infty}^{-\varepsilon} \frac{V(x)}{x^{2}} f(x) d x+\int_{+\varepsilon}^{\infty} \frac{V(x)}{x^{2}} f(x) d x-\frac{2}{\varepsilon} V(0) f(0)\right)
\end{aligned}
$$

for any polynomial $f$ and where the function $V$ represents the regular form $v$

$$
\langle v, f\rangle=\int_{-\infty}^{\infty} V(x) f(x) d x .
$$

The form $v$ is regular if a sequence $\left\{S_{n}\right\}_{n \geq 0}$ exists such that [4]

$$
\begin{array}{cc}
\left\langle v, S_{n} S_{m}\right\rangle=k_{n} \delta_{n, m}, \quad n, m \geq 0, \quad k_{n} \neq 0, & n \geq 0, \\
S_{0}(x)=1, \quad S_{1}(x)=x-\zeta_{0}, & \\
S_{n+2}(x)=\left(x-\zeta_{n+1}\right) S_{n+1}(x)-\rho_{n+1} S_{n}(x), & n \geq 0 .
\end{array}
$$

In algebraic terms, the problem under consideration is the following: determine regular solutions $u$ of the equation

$$
x^{2} u=-\lambda v, \quad \lambda \neq 0,
$$

with constraints $(u)_{0}=1,(v)_{0}=1$ where $(u)_{n}:=\left\langle u, x^{n}\right\rangle, n \geq 0$, and $\left\langle x^{2} u, f\right\rangle:=$ $\left\langle u, x^{2} f(x)\right\rangle$. Equation (1.3) is equivalent to

$$
u=\delta-(u)_{1} \delta^{\prime}-\lambda x^{-2} v
$$

where $\langle\delta, f\rangle=f(0)$; for a form $w, w^{\prime}=D w$ is the derivative: $\left\langle w^{\prime}, f\right\rangle:=-\left\langle w, f^{\prime}\right\rangle$ and $\left\langle x^{-1} v, f\right\rangle:=\left\langle v, \theta_{0} f\right\rangle=\left\langle v, \frac{f(x)-f(0)}{x}\right\rangle$. The second member of (1.4) is the usual sum of a suitable solution of the homogeneous equation and a particular solution of (1.3), since $x^{2}\left(x^{-2} v\right)=v$ following the definitions.

Received February 6, 1995, revised July 20, 1995.

1991 Mathematics Subject Classification. $42 \mathrm{C} 05$.

Key words and phrases: orthogonal polynomials, semi-classical forms, representations. 
When $v$ is given by (1.1), in view of (1.4), we may write

$$
\begin{aligned}
\langle u, f\rangle=f(0) & \left\{1+\lambda P f \int_{-\infty}^{\infty} \frac{V(x)}{x^{2}} d x\right\} \\
+ & f^{\prime}(0)\left\{(u)_{1}+\lambda P \int_{-\infty}^{\infty} \frac{V(x)}{x} d x\right\}-\lambda P f \int_{-\infty}^{\infty} \frac{V(x)}{x^{2}} f(x) d x
\end{aligned}
$$

where

$$
P \int_{-\infty}^{\infty} \frac{V(x)}{x} d x=\lim _{\varepsilon \rightarrow+0}\left(\int_{-\infty}^{-\varepsilon} \frac{V(x)}{x} d x+\int_{+\varepsilon}^{\infty} \frac{V(x)}{x} d x\right) .
$$

We have assumed that $\operatorname{Pf} \int_{-\infty}^{\infty} \frac{V(x)}{x^{2}} d x$ exists. When this number is different from zero, what is the regularity of the form $P f \frac{V(x)}{x^{2}}$ ? We may choose the parameters $(u)_{1}$ and $\lambda$ by the conditions

$$
\begin{gathered}
(u)_{1}=-\lambda P \int_{-\infty}^{\infty} \frac{V(x)}{x} d x \\
\lambda=-\left(\operatorname{Pf} \int_{-\infty}^{\infty} \frac{V(x)}{x^{2}} d x\right)^{-1} .
\end{gathered}
$$

Then, by (1.5)

$$
\langle u, f\rangle=-\lambda P f \int_{-\infty}^{\infty} \frac{V(x)}{x^{2}} f(x) d x
$$

is the solution of (1.3) which we are interested in.

When $\operatorname{Pf} \int_{-\infty}^{\infty} \frac{V(x)}{x^{2}} d x=0$, we maintain (1.6), but now the parameter $\lambda$ becomes arbitrary, and we have by (1.5)

$$
u=\delta-\lambda P f \frac{V(x)}{x^{2}} .
$$

In general, the form $u$ given by (1.4) is regular if and only if $\Delta_{n} \neq 0, n \geq 0[16,(1.12)]$, with

$$
\Delta_{n}=\left\langle v, S_{n}^{2}\right\rangle\left\{\lambda+\sum_{\nu=0}^{n} \frac{\left(\lambda S_{\nu-1}^{(1)}(0)-(u)_{1} S_{\nu}(0)\right)^{2}}{\left\langle v, S_{\nu}^{2}\right\rangle}\right\}, \quad n \geq 0 .
$$

As usual, $S_{n}^{(1)}(x)=\left\langle v, \frac{S_{n+1}(x)-S_{n+1}(\xi)}{x-\xi}\right\rangle, n \geq 0$. In this case, the orthogonal sequence $\left\{Z_{n}\right\}_{n \geq 0}$ relative to $u$ is given by

$$
\begin{aligned}
& Z_{0}(x)=1, \quad Z_{1}(x)=S_{1}(x)+b_{0} \\
& Z_{n+2}(x)=S_{n+2}(x)+b_{n+1} S_{n+1}(x)+a_{n} S_{n}(x), \quad n \geq 0,
\end{aligned}
$$

where by [16, Lemma 1.1]

$$
\begin{gathered}
b_{0}=\zeta_{0}-(u)_{1}, \\
b_{n+1}=\zeta_{n+1}-\frac{\left((u)_{1} S_{n}(0)-\lambda S_{n-1}^{(1)}(0)\right)\left((u)_{1} S_{n+1}(0)-\lambda S_{n}^{(1)}(0)\right)}{\Delta_{n}}, \quad n \geq 0 \\
a_{n}=\frac{\Delta_{n+1}}{\Delta_{n}}, \quad n \geq 0 .
\end{gathered}
$$

Also, the sequence $\left\{Z_{n}\right\}_{n \geq 0}$ satisfies the recurrence relation

$$
\begin{aligned}
& Z_{0}(x)=1, \quad Z_{1}(x)=x-\beta_{0}, \\
& Z_{n+2}(x)=\left(x-\beta_{n+1}\right) Z_{n+1}(x)-\gamma_{n+1} Z_{n}(x), \quad n \geq 0,
\end{aligned}
$$


with

$$
\begin{array}{ll}
\beta_{0}=(u)_{1}, \quad \beta_{n+1}=\zeta_{n+1}+b_{n}-b_{n+1}, \quad n \geq 0, \\
\gamma_{1}=-\Delta_{0}, \quad \gamma_{2}=\lambda \frac{\Delta_{1}}{\Delta_{0}^{2}}, \quad \gamma_{n+3}=\frac{\Delta_{n+2} \Delta_{n}}{\Delta_{n+1}^{2}} \rho_{n+1}, \quad n \geq 0 .
\end{array}
$$

In particular, when $\lambda>0$ and $v$ is positive definite, then $u$ is regular by [16, Theorem 1.1].

When $v$ is symmetric, we have (compare [16])

$$
\begin{gathered}
a_{2 n}=\frac{\lambda \Lambda_{n}+1}{\lambda \Lambda_{n-1}+1} \rho_{2 n+1} \quad a_{2 n+1}=\rho_{2 n+2}, \quad n \geq 0, \\
\gamma_{1}=-\lambda, \quad \gamma_{2 n+2}=a_{2 n}, \quad \gamma_{2 n+3}=\frac{\rho_{2 n+1} \rho_{2 n+2}}{a_{2 n}}, \quad n \geq 0,
\end{gathered}
$$

with

$$
\Lambda_{-1}=0, \quad \Lambda_{n}=\sum_{\nu=0}^{n}\left(\prod_{\mu=0}^{\nu} \frac{\rho_{2 \mu}}{\rho_{2 \mu+1}}\right), \quad n \geq 0, \quad \rho_{0}=1 .
$$

Our aim is to give examples of semi-classical forms (1.8) or (1.9) through data of semi-classical forms $v$.

\section{The structure relation}

In the sequel, the form $v$ will be supposed symmetric, semi-classical of class $\tilde{s}$, and satisfying

$$
D(\widetilde{\Phi} v)+\tilde{\psi} v=0
$$

with $\tilde{s}=\max (\operatorname{deg} \widetilde{\Phi}-2, \operatorname{deg} \tilde{\psi}-1)[14$, Definition 3.1]. The form $u$ given by (1.4) satisfies the equation

$$
D\left(\Phi_{0} u\right)+\psi_{0} u=0
$$

with

$$
\Phi_{0}(x)=x^{2} \widetilde{\Phi}(x), \quad \psi_{0}(x)=x^{2} \tilde{\psi}(x) .
$$

The class of $u$ is at most $\tilde{s}+2$. Following a standard criterion [14, Proposition 3.5], we have

$$
\Phi_{0}^{\prime}(0)+\psi_{0}(0)=0 ; \quad\left\langle u, \vartheta_{0} \psi_{0}+\vartheta_{0}^{2} \Phi_{0}\right\rangle=\widetilde{\Phi}(0)-\lambda\left\langle v, \vartheta_{0} \tilde{\psi}+\vartheta_{0}^{2} \widetilde{\Phi}\right\rangle
$$

If $\widetilde{\Phi}(0)-\lambda\left\langle v, \vartheta_{0} \tilde{\psi}+\vartheta_{0}^{2} \widetilde{\Phi}\right\rangle \neq 0$, then the class is $\tilde{s}+2$.

If $\widetilde{\Phi}(0)-\lambda\left\langle v, \vartheta_{0} \tilde{\psi}+\vartheta_{0}^{2} \widetilde{\Phi}\right\rangle=0$ and $\widetilde{\Phi}(0) \neq 0$, then the class is $\tilde{s}+1$.

Indeed, $u$ satisfies $D(\Phi u)+\psi u=0$ with

$$
\Phi(x)=x \widetilde{\Phi}(x), \quad \psi(x)=\widetilde{\Phi}(x)+x \tilde{\psi}(x) .
$$

Moreover, we have $\Phi^{\prime}(0)+\psi(0)=2 \widetilde{\Phi}(0) \neq 0$. Below, the class will be determined in any particular case. 13]

In order to obtain the structure relation of $\left\{Z_{n}\right\}_{n \geq 0}$, let be the one of $\left\{S_{n}\right\}_{n \geq 0}[9$,

$$
\widetilde{\Phi}(x) S_{n+1}^{\prime}(x)=\frac{1}{2}\left(\widetilde{C}_{n+1}(x)-\widetilde{C}_{0}(x)\right) S_{n+1}(x)-\rho_{n+1} \widetilde{D}_{n+1}(x) S_{n}(x), \quad n \geq 0 .
$$


By (1.2), note that $\zeta_{n}=0$; by symmetry and $n \rightarrow n-1$, we have

$$
\widetilde{\Phi}(x) S_{n}^{\prime}(x)=\widetilde{D}_{n}(x) S_{n+1}(x)+\left(\frac{1}{2}\left(\widetilde{C}_{n}(x)-\widetilde{C}_{0}(x)\right)-x \widetilde{D}_{n}(x)\right) S_{n}(x), \quad n \geq 0 .
$$

From (1.11), using the fact that $b_{n+1}=0$

$$
\widetilde{\Phi}(x) Z_{n+2}^{\prime}(x)=\widetilde{\Phi}(x) S_{n+2}^{\prime}(x)+a_{n} \widetilde{\Phi}(x) S_{n}^{\prime}(x), \quad n \geq 0 .
$$

With (2.6), (2.7), and (1.2), we obtain

$$
\begin{aligned}
& \widetilde{\Phi}(x) Z_{n+2}^{\prime}(x)=\left\{\frac{x\left(\widetilde{C}_{n+2}(x)-\widetilde{C}_{0}(x)\right)}{2}+a_{n} \widetilde{D}_{n}(x)-\rho_{n+2} \widetilde{D}_{n+2}(x)\right\} S_{n+1}(x) \\
& +\left\{a_{n}\left\{\frac{\widetilde{C}_{n}(x)-\widetilde{C}_{0}(x)}{2}-x \widetilde{D}_{n}(x)\right\}-\frac{\widetilde{C}_{n+2}(x)-\widetilde{C}_{0}(x)}{2} \rho_{n+1}\right\} S_{n}(x), \quad n \geq 0 .
\end{aligned}
$$

But, from (1.11) and (1.2), we have

$$
\begin{aligned}
x S_{n+1}(x)+\left(a_{n}-\rho_{n+1}\right) S_{n}(x) & =Z_{n+2}(x), \\
\left(x^{2}+a_{n+1}-\rho_{n+2}\right) S_{n+1}(x)-\rho_{n+1} x S_{n}(x) & =Z_{n+3}(x), \quad n \geq 0,
\end{aligned}
$$

or equivalently, with (1.14),

$$
\begin{aligned}
a_{n} x^{2} S_{n+1}(x) & =a_{n} x Z_{n+2}(x)+\gamma_{n+2}\left(\rho_{n+1}-a_{n}\right) Z_{n+1}(x) \\
a_{n} x^{2} S_{n}(x) & =\left(a_{n+1}-\rho_{n+2}\right) Z_{n+2}(x)+\gamma_{n+2} x Z_{n+1}(x), \quad n \geq 0 .
\end{aligned}
$$

So if we multiply (2.8) by $a_{n} x^{2}$

$$
\begin{aligned}
& a_{n} x^{2} \widetilde{\Phi}(x) Z_{n+2}^{\prime}(x)=\left\{a_{n} x G(x ; n)+\left(a_{n+1}-\rho_{n+2}\right) H(x ; n)\right\} Z_{n+2}(x) \\
& \quad+\left\{-\gamma_{n+2}\left(a_{n}-\rho_{n+1}\right) G(x ; n)+\gamma_{n+2} x H(x ; n)\right\} Z_{n+1}(x), \quad n \geq 0
\end{aligned}
$$

where

$$
\begin{aligned}
& G(x ; n)=\frac{1}{2}\left(\widetilde{C}_{n+2}(x)-\widetilde{C}_{0}(x)\right) x+a_{n} \widetilde{D}_{n}(x)-\rho_{n+2} \widetilde{D}_{n+2}(x), \\
& H(x ; n)=-\frac{1}{2}\left(\widetilde{C}_{n+2}(x)-\widetilde{C}_{0}(x)\right) \rho_{n+1}+a_{n}\left\{\frac{1}{2}\left(\widetilde{C}_{n}(x)-\widetilde{C}_{0}(x)\right)-x \widetilde{D}_{n}(x)\right\}, \quad n \geq 0 .
\end{aligned}
$$

Taking into account the well-known recurrence relations

$$
\begin{aligned}
\widetilde{C}_{n+1}(x) & =-\widetilde{C}_{n}(x)+2 x \widetilde{D}_{n}(x) \\
\rho_{n+2} \widetilde{D}_{n+2}(x) & =-\widetilde{\Phi}(x)+\rho_{n+1} \widetilde{D}_{n}(x)-x \widetilde{C}_{n+1}(x)+x^{2} \widetilde{D}_{n+1}(x), \quad n \geq 0,
\end{aligned}
$$

we obtain

$$
\begin{aligned}
& H(x ; n)=\frac{1}{2}\left(\widetilde{C}_{n+1}(x)+\widetilde{C}_{0}(x)\right)\left(\rho_{n+1}-a_{n}\right)-\rho_{n+1} x \widetilde{D}_{n+1}(x) \\
& G(x ; n)=-\frac{1}{2}\left(\widetilde{C}_{n+1}(x)+\widetilde{C}_{0}(x)\right) x+a_{n} \widetilde{D}_{n}(x)-\rho_{n+2} \widetilde{D}_{n+2}(x)+x^{2} \widetilde{D}_{n+1}(x), \quad n \geq 0 .
\end{aligned}
$$


Moreover, since by $(1.16),\left(a_{n+1}-\rho_{n+2}\right)\left(\rho_{n+1}-a_{n}\right)=0, n \geq 0$, the relation $(2.9)$ becomes

$$
\begin{aligned}
a_{n} x^{2} \widetilde{\Phi}(x) Z_{n+2}^{\prime}(x)=\left\{a_{n} x\left\{\widetilde{\Phi}(x)+\left(a_{n}-\rho_{n+1}\right) \widetilde{D}_{n}(x)+\frac{1}{2}\left(\widetilde{C}_{n+1}(x)-\widetilde{C}_{0}(x)\right) x\right\}\right. \\
\left.-\rho_{n+1}\left(a_{n+1}-\rho_{n+2}\right) x \widetilde{D}_{n+1}(x)\right\} Z_{n+2}(x) \\
-\gamma_{n+2}\left\{\left(a_{n}-\rho_{n+1}\right)\left(a_{n} \widetilde{D}_{n}(x)-a_{n+1} \widetilde{D}_{n+2}\right)+a_{n} x^{2} \widetilde{D}_{n+1}(x)\right\} Z_{n+1}(x) .
\end{aligned}
$$

Finally, when the class is $\tilde{s}+2$, we obtain

$$
\begin{gathered}
x^{2} \widetilde{\Phi}(x) Z_{n+2}^{\prime}(x)=x\left\{\widetilde{\Phi}(x)+\left(a_{n}-\rho_{n+1}\right) \widetilde{D}_{n}(x)+\frac{1}{2}\left(\widetilde{C}_{n+1}(x)-\widetilde{C}_{0}(x)\right) x\right. \\
\left.-\frac{\rho_{n+1}}{a_{n}}\left(a_{n+1}-\rho_{n+2}\right) \widetilde{D}_{n+1}(x)\right\} Z_{n+2}(x) \\
-\gamma_{n+2}\left\{x^{2} \widetilde{D}_{n+1}(x)+\left(a_{n}-\rho_{n+1}\right)\left(\widetilde{D}_{n}(x)-\frac{a_{n+1}}{a_{n}} \widetilde{D}_{n+2}(x)\right)\right\} Z_{n+1}(x), \quad n \geq 0 .
\end{gathered}
$$

When the class is $\tilde{s}+1$, we have

$$
\begin{gathered}
x \widetilde{\Phi}(x) Z_{n+2}^{\prime}(x)=\left\{\widetilde{\Phi}(x)+\left(a_{n}-\rho_{n+1}\right) \widetilde{D}_{n}(x)+\frac{1}{2}\left(\widetilde{C}_{n+1}(x)-\widetilde{C}_{0}(x)\right) x\right. \\
\left.-\frac{\rho_{n+1}}{a_{n}}\left(a_{n+1}-\rho_{n+2}\right) \widetilde{D}_{n+1}(x)\right\} Z_{n+2}(x) \\
-\gamma_{n+2}\left\{x \widetilde{D}_{n+1}(x)+\left(a_{n}-\rho_{n+1}\right)\left(\left(\theta_{0} \widetilde{D}_{n}\right)(x)-\frac{a_{n+1}}{a_{n}}\left(\theta_{0} \widetilde{D}_{n+2}\right)(x)\right)\right\} Z_{n+1}(x),
\end{gathered}
$$

$n \geq 0$.

\section{Examples}

Example 1. We take $v=\mathcal{U}$ where $\mathcal{U}$ is a Tchebychev form of the second kind

$$
\langle\mathcal{U}, f\rangle=\frac{2}{\pi} \int_{-1}^{1} \sqrt{1-x^{2}} f(x) d x .
$$

Thus we have

$$
\begin{array}{rrr}
\rho_{n+1}=\frac{1}{4}, \quad n \geq 0 \quad \text { and } & \lambda=\frac{1}{2}, \\
\widetilde{\Phi}(x)=x^{2}-1, \quad \tilde{\psi}(x)=-3 x, & \tilde{s}=0, \\
\widetilde{C}_{n}(x)=(2 n+1) x, \quad \widetilde{D}_{n}(x)=2(n+1), & n \geq 0 .
\end{array}
$$

Therefore, from (1.16), (1.17), and (2.4)

$$
\begin{array}{rlr}
a_{2 n}=\frac{1}{4} \frac{2 n+3}{2 n+1}, \quad a_{2 n+1}=\frac{1}{4}, & n \geq 0, \\
\gamma_{1}=-\frac{1}{2}, \quad \gamma_{2 n+2}=\frac{1}{4} \frac{2 n+3}{2 n+1}, \quad \gamma_{2 n+3}=\frac{1}{4} \frac{2 n+1}{2 n+3}, & n \geq 0, \\
\Phi(x)=x\left(x^{2}-1\right), \quad \psi(x)=-\left(2 x^{2}+1\right), & s=1 .
\end{array}
$$


From (2.12), we have

$$
\begin{aligned}
& x\left(x^{2}-1\right) Z_{2 n+2}^{\prime}(x)=2(n+1) x^{2} Z_{2 n+2}(x)-(n+1) \frac{2 n+3}{2 n+1} x Z_{2 n+1}(x), \\
& x\left(x^{2}-1\right) Z_{2 n+3}^{\prime}(x)=\left((2 n+3) x^{2}-2\right) Z_{2 n+3}(x)-\frac{1}{2}(2 n+1) x Z_{2 n+2}(x),
\end{aligned}
$$

$n \geq 0$. We deduce the fact that $Z_{n+1}(x)$ has simple zeros. Indeed, on account of (3.3) and (3.4), the equations $Z_{n+1}(x)=0, Z_{n+1}^{\prime}(x)=0$ imply $x=0$; furthermore, we have from (3.4)

$$
Z_{2 n+3}^{\prime}(0)=-\frac{1}{2}(2 n+1) Z_{2 n+2}(0) \neq 0, \quad n \geq 0 .
$$

In fact, the form $u$ is equal to the inverse of the Tchebychev form of the first kind (also see [8]): $u=\mathcal{T}^{-1}$ where

$$
\langle\mathcal{T}, f\rangle=\frac{1}{\pi} \int_{-1}^{1} \frac{f(x)}{\sqrt{1-x^{2}}} d x .
$$

The inverse $u^{-1}$ of $u$ is defined by $u^{-1} u=u u^{-1}=\delta$ with $\langle\delta, f\rangle=f(0)$ and the product $u v$ of two forms is given by [11]

$$
\langle u v, f\rangle:=\langle u,(v f)(x)\rangle=\left\langle u,\left\langle v, \frac{\xi f(\xi)-x f(x)}{\xi-x}\right\rangle\right\rangle .
$$

Indeed, we have $x^{2} u=-\frac{1}{2} \mathcal{U}$. But if $\mathcal{T}^{(1)}$ denotes the form associated with $\mathcal{T}$, we have by [13] that $\frac{1}{2} \mathcal{T}^{(1)}=-x^{2} \mathcal{T}^{-1}$ and the well-known property $\mathcal{T}^{(1)}=\mathcal{U}$, therefore $x^{2}\left(u-\mathcal{T}^{-1}\right)=0$ which implies $u=\mathcal{T}^{-1}$. Thus (see [16, Introduction] for another expression)

$$
\mathcal{T}^{-1}=-\frac{1}{\pi} P f \frac{Y\left(1-x^{2}\right) \sqrt{1-x^{2}}}{x^{2}}
$$

where $Y$ is the characteristic function of $R^{+}$.

Let us consider the quadratic decomposition of $\left\{S_{n}\right\}_{n \geq 0}$ and $\left\{Z_{n}\right\}_{n \geq 0}[4,12]$

$$
\begin{aligned}
& S_{2 n}(x)=\widetilde{P}_{n}\left(x^{2}\right), \quad S_{2 n+1}(x)=x \widetilde{R}_{n}\left(x^{2}\right), \quad n \geq 0, \\
& Z_{2 n}(x)=P_{n}\left(x^{2}\right), \quad Z_{2 n+1}(x)=x R_{n}\left(x^{2}\right), \quad n \geq 0 \text {. }
\end{aligned}
$$

The sequences $\left\{P_{n}\right\}_{n \geq 0}$ and $\left\{R_{n}\right\}_{n \geq 0}$ respectively are orthogonal with respect to $\sigma u$ and $x \sigma u$ where $\sigma u$ is the even part of $u$ defined by $\langle\sigma u, f\rangle:=\langle u,(\sigma f)(x)\rangle=\left\langle u, f\left(x^{2}\right)\right\rangle$. From (1.3), we have

$$
\sigma\left(x^{2} u\right)=x \sigma u=-\lambda \sigma \mathcal{U}=-\frac{1}{2} \sigma \mathcal{U} .
$$

Therefore, $R_{n}=\widetilde{P}_{n}, n \geq 0$. On the other hand

$$
\langle\sigma \mathcal{U}, f\rangle=\frac{2}{\pi} \int_{0}^{1} \sqrt{\frac{1-x}{x}} f(x) d x .
$$

We conclude that $Z_{2 n+1}$ has (simple) zeros in ]-1, $+1[$.

From (3.8) and (3.9), we have

$$
\begin{gathered}
\sigma u=\delta-\frac{1}{2} x^{-1} \sigma \mathcal{U} \\
\langle\sigma u, f\rangle=f(0)-\frac{1}{\pi} \int_{0}^{1} \sqrt{\frac{1-x}{x}} \frac{f(x)-f(0)}{x} d x .
\end{gathered}
$$


With the definition

we obtain

$$
\operatorname{Pf} \int_{0}^{1} \frac{\sqrt{1-x}}{x^{3 / 2}} f(x) d x:=\lim _{\varepsilon \rightarrow+0}\left\{\int_{\varepsilon}^{1} \frac{\sqrt{1-x}}{x^{3 / 2}} f(x) d x-\frac{2}{\sqrt{\varepsilon}} f(0)\right\}
$$

$$
\langle\sigma u, f\rangle=f(0)\left(1+P f \frac{1}{\pi} \int_{0}^{1} \frac{\sqrt{1-x}}{x^{3 / 2}} d x\right)-P f \frac{1}{\pi} \int_{0}^{1} \frac{\sqrt{1-x}}{x^{3 / 2}} f(x) d x .
$$

But

$$
P f \frac{1}{\pi} \int_{0}^{1} \frac{\sqrt{1-x}}{x^{3 / 2}} d x=-\frac{1}{\pi} \int_{0}^{1} \frac{d x}{\sqrt{x(1-x)}}=-1
$$

Therefore,

$$
\sigma \mathcal{T}^{-1}=-P f \frac{1}{\pi} \frac{Y(x(1-x)) \sqrt{1-x}}{x^{3 / 2}}
$$

Thus $\left\{R_{n}\right\}_{n \geq 0}$ and $\left\{P_{n}\right\}_{n \geq 0}$ are orthogonal with respect to the forms (3.9) and (3.10), respectively.

Example 2. We take $v=\mathcal{T}$ or $V(x)=\frac{1}{\pi} \frac{Y\left(1-x^{2}\right)}{\sqrt{1-x^{2}}}$. Here, we have $\operatorname{Pf} \int_{-\infty}^{\infty} \frac{V(x)}{x^{2}} d x=0$, consequently, the form $u$ is given by (1.9) where $\lambda$ is arbitrary. Then

$$
u=\delta-\lambda P f \frac{1}{\pi} \frac{Y\left(1-x^{2}\right)}{x^{2} \sqrt{1-x^{2}}} .
$$

Thus, we have

$$
\begin{gathered}
\rho_{n+1}=\frac{1}{4^{1-\delta_{n, 0} / 2}}, \quad n \geq 0, \\
\widetilde{\Phi}(x)=x^{2}-1, \quad \tilde{\psi}(x)=-x, \quad \tilde{s}=0, \\
\widetilde{C}_{n}(x)=(2 n-1) x, \quad \widetilde{D}_{n}(x)=2 n, \quad n \geq 0 .
\end{gathered}
$$

Therefore, from (1.16), (1.17), and (2.3)

$$
\begin{aligned}
a_{2 n} & =\frac{1}{4^{1-\delta_{n, 0} / 2}} \frac{2(n+1) \lambda+1}{2 n \lambda+1}, \quad a_{2 n+1}=\frac{1}{4}, \quad n \geq 0, \\
\gamma_{1} & =-\lambda, \quad \gamma_{2 n+2}=a_{2 n}, \quad \gamma_{2 n+3}=\frac{1}{4} \frac{2 n \lambda+1}{2(n+1) \lambda+1}, \quad n \geq 0, \\
\Phi(x) & =x^{2}\left(x^{2}-1\right), \quad \psi(x)=-x^{3}, \quad s=2 .
\end{aligned}
$$

The form $u$ is regular if and only if $\lambda \neq-\frac{1}{2 n}, n \geq 1$. From (2.11), we have

$$
\begin{aligned}
& x^{2}\left(x^{2}-1\right) Z_{2 n+2}^{\prime}(x)=x\left\{2(n+1) x^{2}-\frac{1}{2 n \lambda+1}\right\} Z_{2 n+2}(x) \\
&-2 \gamma_{2 n+2}\left\{(2 n+1) x^{2}-\frac{\lambda}{(2 n \lambda+1)(2(n+1) \lambda+1)}\right\} Z_{2 n+1}(x), \\
& x^{2}\left(x^{2}-1\right) Z_{2 n+3}^{\prime}(x)=x\left\{(2 n+3) x^{2}-\frac{4(n+1) \lambda+1}{2(n+1) \lambda+1}\right\} Z_{2 n+3}(x) \\
&-4(n+1) \gamma_{2 n+3} x^{2} Z_{2 n+2}(x),
\end{aligned}
$$

$n \geq 0$. We deduce from $(3.14)$

$$
\frac{\lambda}{2(n+1) \lambda+1} Z_{2 n+3}^{\prime}(0)=-2 \gamma_{2 n+3} Z_{2 n+2}(0) \neq 0, \quad n \geq 0 .
$$


So, the polynomials $Z_{2 n+3}(x)$ have simple zeros. About $Z_{2 n+2}(x)$, we have the result: a multiple root $x$ necessarily satisfies

$$
(2 n+1) x^{2}=\frac{\lambda}{(2 n \lambda+1)(2(n+1) \lambda+1)} .
$$

With the recurrences relations (2.10) for $C_{n}, D_{n}$, the relations (3.13) and (3.14) give

$$
\begin{aligned}
& C_{2 n+1}(x)=x\left\{(4 n-1) x^{2}-\frac{4 n \lambda}{2 n \lambda+1}\right\}, \quad n \geq 0, \\
& C_{2 n+2}(x)=x\left\{(4 n+1) x^{2}+\frac{4 n \lambda}{2 n \lambda+1}\right\}, \quad n \geq 0 . \\
& D_{2 n+1}(x)=4 n x^{2}, \quad n \geq 0, \\
& D_{2 n+2}(x)=2(2 n+1) x^{2}-\frac{2 \lambda}{(2 n \lambda+1)(2(n+1) \lambda+1)}, \quad n \geq 0 .
\end{aligned}
$$

The decomposition (3.7) shows that $R_{n}=\widetilde{P}_{n}, n \geq 0$ since $x \sigma u=-\lambda \sigma \mathcal{T}$. Consequently, the polynomial $Z_{2 n+1}$ does not depend on $\lambda$. Moreover

$$
\langle\sigma \mathcal{T}, f\rangle=\frac{1}{\pi} \int_{0}^{1} \frac{f(x)}{\sqrt{x(1-x)}} d x .
$$

This implies that the roots of $Z_{2 n+1}$ are in $]-1,+1[$. On the other hand, from $\sigma u=$ $\delta-\lambda x^{-1} \sigma \mathcal{T}$, we obtain

$$
\begin{aligned}
\langle\sigma u, f\rangle & =f(0)-\lambda \frac{1}{\pi} \int_{0}^{1} \frac{1}{\sqrt{x(1-x)}} \frac{f(x)-f(0)}{x} d x \\
& =\left\{1+\lambda P f \frac{1}{\pi} \int_{0}^{1} \frac{1}{x^{3 / 2}} \frac{d x}{\sqrt{1-x}}\right\} f(0)-\lambda P f \frac{1}{\pi} \int_{0}^{1} \frac{1}{x^{3 / 2}} \frac{f(x)}{\sqrt{1-x}} d x .
\end{aligned}
$$

But $\operatorname{Pf} \frac{1}{\pi} \int_{0}^{1} \frac{1}{x^{3 / 2}} \frac{d x}{\sqrt{1-x}}=0 ;$ therefore,

$$
\sigma u=\delta-\lambda P f \frac{1}{\pi} \frac{Y(x(1-x))}{x^{3 / 2} \sqrt{1-x}} .
$$

Thus $\left\{R_{n}\right\}_{n \geq 0}$ and $\left\{P_{n}\right\}_{n \geq 0}$ are orthogonal with respect to the forms (3.17) and (3.18), respectively. In Examples 1 and 2, the form $u$ is a second-degree form, since $v$ is a second-degree form $[15,17,18]$. Indeed, if $S(u)(z)=-\sum_{n \geq 0} \frac{(u)_{n}}{z^{n+1}}$ denotes its formal Stieltjes function, we have using $[15,(4.1)$ and (4.15)]

$$
\begin{gathered}
z^{2} S(u)(z)+z=-\lambda S(v)(z), \\
\widetilde{B}(z) S^{2}(v)(z)+\widetilde{C}(z) S(v)(z)+\widetilde{D}(z)=0,
\end{gathered}
$$

where in Example 1,

$$
\widetilde{B}(z)=1, \quad \widetilde{C}(z)=4 z, \quad \widetilde{D}(z)=4,
$$

and in Example 2,

$$
\widetilde{B}(z)=z^{2}-1, \quad \widetilde{C}(z)=0, \quad \widetilde{D}(z)=-1 .
$$

Therefore, $S(u)(z)$ satisfies

$$
B(z) S^{2}(u)(z)+C(z) S(u)(z)+D(z)=0
$$


with

$$
\begin{aligned}
& B(z)=z^{4} \widetilde{B}(z) \\
& C(z)=z^{2}\{2 z \widetilde{B}(z)-\lambda \widetilde{C}(z)\} \\
& D(z)=z^{2} \widetilde{B}(z)-\lambda z \widetilde{C}(z)+\lambda^{2} \widetilde{D}(z) .
\end{aligned}
$$

Moreover, the form $u$ also satisfies [15, Proposition 2.3]

$$
B(x) u+x^{2} D(x) u^{-1}=0 .
$$

Example 3. We take $v=\mathcal{H}$ where $\mathcal{H}$ denotes the Hermite form, then $V(x)=\frac{1}{\sqrt{\pi}} e^{-x^{2}}$. Here

$$
\begin{gathered}
\rho_{n+1}=\frac{1}{2}(n+1), \quad n \geq 0, \quad \lambda=\frac{1}{2}, \\
\widetilde{\Phi}(x)=1, \quad \tilde{\psi}(x)=2 x, \quad \tilde{s}=0, \\
\widetilde{C}_{n}(x)=-2 x, \quad \widetilde{D}_{n}(x)=-2, \quad n \geq 0 .
\end{gathered}
$$

Then, from (1.16), (1.17), and (2.4)

$$
\begin{gathered}
a_{2 n}=n+1, \quad a_{2 n+1}=n+1, \quad n \geq 0, \\
\gamma_{n+1}=\frac{1}{2}\left(n+(-1)^{n+1}\right), \quad n \geq 0, \\
\Phi(x)=x, \quad \psi(x)=2 x^{2}+1, \quad s=1 .
\end{gathered}
$$

In fact, the form given by (1.8) is the generalized Hermite form corresponding to the parameter value $\mu=-1$ [4]. We have

$$
C_{n}(x)=-2\left(x^{2}+(-1)^{n}\right), \quad D_{n}(x)=-2 x, \quad n \geq 0 .
$$

Hence

$$
x Z_{n+2}^{\prime}(x)=\left(1+(-1)^{n+1}\right) Z_{n+2}(x)+2 \gamma_{n+2} x Z_{n+1}(x), \quad n \geq 0 .
$$

The roots of $Z_{n+2}$ are simple since

$$
Z_{2 n+3}^{\prime}(0)=-2 \gamma_{2 n+3} Z_{2 n+2}(0) \neq 0, \quad n \geq 0 .
$$

From the decomposition (3.7), we have $R_{n}=\widetilde{P}_{n}, n \geq 0$, where the sequence $\left\{\widetilde{P}_{n}\right\}_{n \geq 0}$ is a Laguerre sequence with parameter value of $-1 / 2$, since

$$
\langle\sigma \mathcal{H}, f\rangle=\frac{1}{\sqrt{\pi}} \int_{0}^{\infty} x^{-\frac{1}{2}} e^{-x} f(x) d x
$$

Therefore, the roots of $Z_{2 n+1}(x)$ are real. Next, from $\sigma u=\delta-\frac{1}{2} x^{-1} \sigma \mathcal{H}$ and (3.24), we obtain

$$
\langle\sigma u, f\rangle=\left\{1+P f \frac{1}{2 \sqrt{\pi}} \int_{0}^{\infty} \frac{e^{-x}}{x^{3 / 2}} d x\right\} f(0)-P f \frac{1}{2 \sqrt{\pi}} \int_{0}^{\infty} \frac{e^{-x}}{x^{3 / 2}} f(x) d x .
$$

But $P f \frac{1}{2 \sqrt{\pi}} \int_{0}^{\infty} \frac{e^{-x}}{x^{3 / 2}} d x=-1$; therefore,

$$
\langle\sigma u, f\rangle=-P f \frac{1}{2 \sqrt{\pi}} \int_{0}^{\infty} \frac{e^{-x}}{x^{3 / 2}} f(x) d x
$$

So, $\left\{P_{n}\right\}_{n \geq 0}$ is a Laguerre sequence with parameter value of $-3 / 2$.

In these examples, where $v$ is symmetric, positive definite, and $\lambda>0$, we have [11]

$$
u^{(1)}=u^{-1} v \text {, }
$$

and the fact that $u^{(1)}$ and $u^{-1}$ are positive definite. As usual, $u^{(1)}$ is associated with $u$. 
Indeed, from $(1.3),\left(x^{2} u\right) u^{-1}=-\lambda v u^{-1}$. But following the general formula $f(u v)=$ $(f u) v+x\left(u \theta_{0} f\right)(x) v[14,(2.6)]$, we have $\left(x^{2} u\right) u^{-1}=x^{2} \delta-x\left(u \theta_{0} \zeta^{2}\right)(x) u^{-1}=$ $-x\left((u)_{1}+x\right) u^{-1}=-x^{2} u^{-1}$. From the definition $[11,13,(4.7)], \gamma_{1} u^{(1)}=-x^{2} u^{-1}$; therefore, with $\gamma_{1}=-\lambda$, we obtain (3.26). Next, if $\left\{Z_{n}^{(-)}\right\}_{n \geq 0}$ denotes the orthogonal sequence with respect to $u^{-1}$ when it exists, from the general theory we have [13] (compare also [16])

$$
\begin{gathered}
Z_{n+2}^{(-)}(x)=Z_{n+2}^{(1)}(x)+a_{n}^{(-)} Z_{n}^{(1)}(x), \quad n \geq 0, \\
a_{2 n}^{(-)}=-\frac{Z_{2 n+3}^{\prime}(0)}{Z_{2 n+1}^{\prime}(0)}, \quad a_{2 n+1}^{(-)}=\gamma_{2 n+3}, \quad n \geq 0, \\
Z_{2 n+1}^{\prime}(0)=(-1)^{n} \sum_{\nu=0}^{n} \frac{1}{\gamma_{2 \nu} \gamma_{2 \nu+1}}\left(\prod_{\mu=\nu}^{n} \gamma_{2 \mu}\right)\left(\prod_{\mu=0}^{\nu} \gamma_{2 \mu+1}\right), \quad n \geq 0, \\
\gamma_{1}^{(-)}=-\gamma_{1}, \quad \gamma_{2 n+2}^{(-)}=a_{2 n}^{(-)}, \quad \gamma_{2 n+3}^{(-)}=\frac{\gamma_{2 n+2} \gamma_{2 n+3}}{a_{2 n}^{(-)}}, \quad n \geq 0 .
\end{gathered}
$$

The announced properties follow since $Z_{2 n+3}^{\prime}(0) \neq 0, n \geq 0$, from (3.5), (3.15), and (3.23).

Remark. It is a striking property that the product of two positive definite forms is still positive definite. In general, this is not true. For example, the Tchebychev form $\mathcal{T}$ satisfies the equation $\left(x^{2}-1\right) \mathcal{T}^{2}=0$. Consequently, $\mathcal{T}^{2}=\frac{1}{2}\left(\delta_{-1}+\delta_{1}\right)$. Positivity is kept, but no regularity.

As an application of (3.26), we have

$$
u^{(2)}=-\frac{\gamma_{1}}{\gamma_{2}} \delta+\frac{\rho_{1}}{\gamma_{2}} v^{(1)} .
$$

Indeed, we have $\gamma_{1}^{(1)} u^{(2)}=-x^{2}\left(u^{(1)}\right)^{-1}$, and from (3.26), $\left(u^{(1)}\right)^{-1}=v^{-1} u$; therefore, with the help of $[15,(2.6)]$ and $(1.3)$,

$$
\gamma_{1}^{(1)} u^{(2)}=-x^{2}\left(v^{-1} u\right)=-\left(x^{2} u\right) v^{-1}-x(u \zeta)(x) v^{-1}=\lambda \delta-x^{2} v^{-1} .
$$

But $\rho_{1} v^{(1)}=-x^{2} v^{-1}$, hence (3.28) holds. For a general problem analogous to (3.28) and detailed properties, see $[5,10]$.

\section{Representation of $u^{-1}, u^{(1)}, u^{(2)}$ in Examples 1 and 2}

In the case of Example 1, we easily obtain $u^{(1)}=2 x^{2} \mathcal{T}$ by $(3.26)$; therefore,

$$
\left\langle u^{(1)}, f\right\rangle=\frac{2}{\pi} \int_{-1}^{1} \frac{x^{2}}{\sqrt{1-x^{2}}} f(x) d x .
$$

From (3.28)

$$
\left\langle u^{(2)}, f\right\rangle=\frac{2}{3}\langle\delta, f\rangle+\frac{2}{3 \pi} \int_{-1}^{1} \sqrt{1-x^{2}} f(x) d x .
$$

In Example 2, we have $z^{2} S(u)(z)+z=\lambda\left(z^{2}-1\right)^{-1 / 2}$ where we take the branch $\sqrt{z^{2}-1}$ which is positive when $z=x>1$. Writing $z^{2}\left(z^{2}-1\right)-\lambda^{2}=\left(z^{2}-t_{\lambda}^{2}\right)\left(z^{2}-\tau_{\lambda}^{2}\right)$ with $t_{\lambda}^{2}=\frac{1}{2}\left(1+\sqrt{1+4 \lambda^{2}}\right), \tau_{\lambda}^{2}=\frac{1}{2}\left(1-\sqrt{1+4 \lambda^{2}}\right)$, we get, with the help of $[15,(1.5)]$,

$$
S\left(u^{-1}\right)(z)=\frac{1}{z^{2} S(u)(z)}=-\frac{1}{-\lambda\left(z^{2}-1\right)^{-1 / 2}+z}=\frac{\lambda \sqrt{z^{2}-1}+z\left(z^{2}-1\right)}{\left(t_{\lambda}^{2}-z^{2}\right)\left(z^{2}-\tau_{\lambda}^{2}\right)} .
$$


Suppose $\lambda>0$ and let $S\left(u^{-1}\right)(z)=H(z)$. In this case, $\Im H(z)>0$ when $\Im z>0$ $(z=x+i y)$.

Moreover, since

we have

$$
-i y H(i y)=\frac{y}{\lambda\left(1+y^{2}\right)^{-1 / 2}+y} \rightarrow 1, \quad y \rightarrow \infty,
$$

$$
H(z)=\int_{-\infty}^{\infty} \frac{d \mu(t)}{t-z}
$$

where $\mu$ is a non-decreasing function [7]. We see that $\mu$ is continuous except at $t^{2}=t_{\lambda}^{2}$ since

$$
\begin{gathered}
(t-z) H(z) \rightarrow 0, \quad z \rightarrow t, \quad 0<\varepsilon \leq \arg (z-t) \leq \pi-\varepsilon, \quad t \in R, \quad t^{2} \neq t_{\lambda}^{2}, \\
\left( \pm t_{\lambda}-z\right) H(z) \rightarrow \frac{1}{2} \frac{\sqrt{1+4 \lambda^{2}}-1}{\sqrt{1+4 \lambda^{2}}}:=k_{\lambda}, \quad z \rightarrow \pm t_{\lambda}, \quad 0<\varepsilon \leq \arg \left(z- \pm t_{\lambda}\right) \leq \pi-\varepsilon .
\end{gathered}
$$

In fact, the function $\mu$ is absolutely continuous with respect to Lebesgue measure for $t^{2} \neq t_{\lambda}^{2}$ since

$$
\int_{|x|>T}|H(x+i y)|^{2} d x \leq B_{1}, \quad 0<y \leq 1, \quad T>t_{\lambda}>1,
$$

from the definition of $\mathrm{H}$. Moreover, it is easy to see that

$$
\int_{-R}^{R}|H(x+i y)|^{2} d x \leq B_{2}, \quad 0<y \leq \frac{1}{2} t_{\lambda}
$$

with $0<R<t_{\lambda}$, since

$$
\begin{array}{lll}
\left|z^{2}-t_{\lambda}^{2}\right| \geq t_{\lambda}^{2}-R^{2}, & & |x| \leq R, \\
\left|z^{2}-\tau_{\lambda}^{2}\right| \geq \frac{1}{2} \tilde{\tau}_{\lambda}\left(x^{2}+\tilde{\tau}_{\lambda}^{2}\right)^{1 / 2}, & & 0<y \leq \frac{1}{2} \tilde{\tau}_{\lambda}, \quad \tilde{\tau}_{\lambda}=\sqrt{-\tau_{\lambda}^{2}} .
\end{array}
$$

The property follows from the well-known formula

On account of

$$
\mu\left(x_{2}\right)-\mu\left(x_{1}\right)=\lim _{y \rightarrow 0+} \frac{1}{\pi} \int_{x_{1}}^{x_{2}} \Im H(x+i y) d x .
$$

$$
\Im H(x+i 0)= \begin{cases}\frac{\lambda \sqrt{1-x^{2}}}{x^{2}\left(1-x^{2}\right)+\lambda^{2}}, & |x|<1, \\ 0, & |x|>1\end{cases}
$$

we finally obtain

$$
H(z)=k_{\lambda} \frac{2 z}{t_{\lambda}^{2}-z^{2}}+\frac{\lambda}{\pi} \int_{-1}^{1} \frac{\sqrt{1-t^{2}}}{t^{2}\left(1-t^{2}\right)+\lambda^{2}} \frac{d t}{t-z}, \quad z \in C-[-1,+1] .
$$

Consequently,

$$
\left\langle u^{-1}, f\right\rangle=k_{\lambda}\left\langle\delta_{t_{\lambda}}+\delta_{-t_{\lambda}}, f\right\rangle+\frac{\lambda}{\pi} \int_{-1}^{1} \frac{\sqrt{1-x^{2}}}{x^{2}\left(1-x^{2}\right)+\lambda^{2}} f(x) d x, \quad \lambda>0 .
$$

From $\gamma_{1} u^{(1)}=-x^{2} u^{-1}$ and (4.1), we deduce

$$
\left\langle u^{(1)}, f\right\rangle=\frac{\lambda}{\sqrt{1+4 \lambda^{2}}}\left\langle\delta_{t_{\lambda}}+\delta_{-t_{\lambda}}, f\right\rangle+\frac{1}{\pi} \int_{-1}^{1} \frac{x^{2} \sqrt{1-x^{2}}}{x^{2}\left(1-x^{2}\right)+\lambda^{2}} f(x) d x .
$$


The formula (3.28) gives

$$
\left\langle u^{(2)}, f\right\rangle=\frac{2 \lambda}{1+2 \lambda}\langle\delta, f\rangle+\frac{2}{\pi(1+2 \lambda)} \int_{-1}^{1} \sqrt{1-x^{2}} f(x) d x .
$$

Remark. By straightforward and lengthy calculation, it could be shown with the help of equation (3.20) that the representations (4.1) and (4.2) remain valid for $\lambda \in$ $C-\left[-\frac{1}{2} i,+\frac{1}{2} i\right]$.

When $\lambda=-\frac{1}{2 n}, n \geq 1$, the forms $u^{-1}$ and $u^{(1)}$ are no longer regular, but keep their respective representations. As to (4.3), it is valid for $\lambda \neq-\frac{1}{2}$.

It remains to treat the case $\lambda=i \zeta, 0<|\zeta| \leq \frac{1}{2}$ where $0<t_{\lambda}^{2}, \tau_{\lambda}^{2}<1$. It will be sufficient to suppose $0<\zeta \leq \frac{1}{2}$. Equation (3.20) becomes

$$
x^{4}\left(x^{2}-1\right) u+x^{2}\left(x^{2}\left(x^{2}-1\right)+\zeta^{2}\right) u^{-1}=0 .
$$

Equivalently,

$$
x^{2}\left(x^{2}-1\right) u+\left(x^{2}\left(x^{2}-1\right)+\zeta^{2}\right) u^{-1}=A \delta+B \delta^{\prime} .
$$

We deduce that $(u)_{4}-(u)_{2}+\left(u^{-1}\right)_{4}-\left(u^{-1}\right)_{2}+\zeta^{2}=A, 0=B$. But $(u)_{2}=-\lambda$, $(u)_{4}=-\lambda(\mathcal{T})_{2}=-\frac{1}{2} \lambda,\left(u^{-1}\right)_{2}=\lambda,\left(u^{-1}\right)_{4}=\lambda^{2}+\frac{1}{2} \lambda$. Hence, $A=0$. Therefore,

$$
\left(x^{2}-\tau_{\lambda}^{2}\right)\left(x^{2}-t_{\lambda}^{2}\right) u^{-1}=-x^{2}\left(x^{2}-1\right) u=\lambda\left(x^{2}-1\right) \mathcal{T}=-\frac{1}{2} \lambda \mathcal{U} .
$$

The following identity will be very useful: for any polynomial $f$ and any form $w$, we have $[15,(1.12)]$

$$
(x-c)^{-1}(f(x) w)=f(c)\left((x-c)^{-1} w\right)+\left(\theta_{c} f\right)(x) w-\left\langle w, \theta_{c} f\right\rangle \delta_{c}
$$

where $\left(\theta_{c} f\right)(x):=\frac{f(x)-f(c)}{x-c},\left\langle(x-c)^{-1} w, f\right\rangle:=\left\langle w, \theta_{c} f\right\rangle, c \in C$. Two cases arise: (1) $0<\zeta<\frac{1}{2}$, and $(2) \zeta=\frac{1}{2}$.

(1) $0<\zeta<\frac{1}{2}$.

Then $\frac{1}{2}<t_{i \zeta}^{2}<1,0<\tau_{i \zeta}^{2}<\frac{1}{2}$. We have successively, in accordance with (4.4) and $(4.5)$,

$$
\begin{gathered}
\left(x+\tau_{i \zeta}\right)\left(x^{2}-t_{i \zeta}^{2}\right) u^{-1}=\tau_{i \zeta}\left(i \zeta-t_{i \zeta}^{2}\right) \delta_{\tau_{i \zeta}}-\frac{1}{2} i \zeta\left(x-\tau_{i \zeta}\right)^{-1} \mathcal{U} \\
\left(x^{2}-t_{i \zeta}^{2}\right) u^{-1}=\left(i \zeta-t_{i \zeta}^{2}\right) \delta_{-\tau_{i \zeta}}+\tau_{i \zeta}\left(i \zeta-t_{i \zeta}^{2}\right)\left(x+\tau_{i \zeta}\right)^{-1} \delta_{\tau_{i \zeta}}-\frac{1}{2} i \zeta\left(x^{2}-\tau_{i \zeta}^{2}\right)^{-1} \mathcal{U} \\
\left(x-t_{i \zeta}\right) u^{-1}=-t_{i \zeta} \delta_{-t_{i \zeta}}+\tau_{i \zeta}\left(i \zeta-t_{i \zeta}^{2}\right)\left(x+t_{i \zeta}\right)^{-1}\left(x+\tau_{i \zeta}\right)^{-1} \delta_{\tau_{i \zeta}} \\
+\left(i \zeta-t_{i \zeta}^{2}\right)\left(x+t_{i \zeta}\right)^{-1} \delta_{-\tau_{i \zeta}}-\frac{1}{2} i \zeta\left(x+t_{i \zeta}\right)^{-1}\left(x^{2}-\tau_{i \zeta}^{2}\right)^{-1} \mathcal{U} \\
u^{-1}=\delta_{t_{i \zeta}}-t_{i \zeta}\left(x-t_{i \zeta}\right)^{-1} \delta_{-t_{i \zeta}}+\tau_{i \zeta}\left(i \zeta-t_{i \zeta}^{2}\right)\left(x^{2}-t_{i \zeta}^{2}\right)^{-1}\left(x+\tau_{i \zeta}\right)^{-1} \delta_{\tau_{i \zeta}} \\
+\left(i \zeta-t_{i \zeta}^{2}\right)\left(x^{2}-t_{i \zeta}^{2}\right)^{-1} \delta_{-\tau_{i \zeta}}-\frac{1}{2} i \zeta\left(x^{2}-t_{i \zeta}^{2}\right)^{-1}\left(x^{2}-\tau_{i \zeta}^{2}\right)^{-1} \mathcal{U}
\end{gathered}
$$


Further, on account of the definitions and of the representation of $\mathcal{U}$, we have

$$
\begin{aligned}
& \left\langle\left(x^{2}-\tau_{i \zeta}^{2}\right)^{-1}\left(x^{2}-t_{i \zeta}^{2}\right)^{-1} \mathcal{U}, f\right\rangle=\left\langle\mathcal{U}, \theta_{t_{i \zeta}} \theta_{-t_{i \zeta}} \theta_{\tau_{i \zeta}} \theta_{-\tau_{i \zeta}} f\right\rangle \\
= & \frac{2}{\pi} \int_{-1}^{1} \sqrt{1-x^{2}} \frac{\left(\theta_{-t_{i \zeta}} \theta_{\tau_{i \zeta}} \theta_{-\tau_{i \zeta}} f\right)(x)-\left(\theta_{-t_{i \zeta}} \theta_{\tau_{i \zeta}} \theta_{-\tau_{i \zeta}} f\right)\left(t_{i \zeta}\right)}{x-t_{i \zeta}} d x \\
= & -\left(\theta_{-t_{i \zeta}} \theta_{\tau_{i \zeta}} \theta_{-\tau_{i \zeta}} f\right)\left(t_{i \zeta}\right) P \frac{2}{\pi} \int_{-1}^{1} \frac{\sqrt{1-x^{2}}}{x-t_{i \zeta}} d x \\
& -\left(\theta_{\tau_{i \zeta}} \theta_{-\tau_{i \zeta}} f\right)\left(-t_{i \zeta}\right) P \frac{2}{\pi} \int_{-1}^{1} \frac{\sqrt{1-x^{2}}}{x^{2}-t_{i \zeta}^{2}} d x \\
& -\left(\theta_{-\tau_{i \zeta}} f\right)\left(\tau_{i \zeta}\right) P \frac{2}{\pi} \int_{-1}^{1} \frac{\sqrt{1-x^{2}}}{\left(x^{2}-t_{i \zeta}^{2}\right)\left(x-\tau_{i \zeta}\right)} d x \\
& -f\left(-\tau_{i \zeta}\right) P \frac{2}{\pi} \int_{-1}^{1} \frac{\sqrt{1-x^{2}} d x}{\left(x^{2}-t_{i \zeta}^{2}\right)\left(x^{2}-\tau_{i \zeta}^{2}\right)}+P \frac{2}{\pi} \int_{-1}^{1} \frac{\sqrt{1-x^{2}} f(x) d x}{\left(x^{2}-t_{i \zeta}^{2}\right)\left(x^{2}-\tau_{i \zeta}^{2}\right)}
\end{aligned}
$$

where $P$ means Cauchy's principal value of the integral. But, it is easy to see that

$$
\begin{gathered}
P \frac{2}{\pi} \int_{-1}^{1} \frac{\sqrt{1-t^{2}}}{t-x} d t=-2 x, \quad P \frac{2}{\pi} \int_{-1}^{1} \frac{\sqrt{1-t^{2}}}{t^{2}-x^{2}} d t=-2, \quad|x|<1 \\
P \frac{2}{\pi} \int_{-1}^{1} \frac{\sqrt{1-t^{2}}}{\left(t^{2}-x_{1}^{2}\right)\left(t-x_{2}\right)} d t=0, \quad P \frac{2}{\pi} \int_{-1}^{1} \frac{\sqrt{1-t^{2}}}{\left(t^{2}-x_{1}^{2}\right)\left(t^{2}-x_{2}^{2}\right)} d t=0 \\
-1<x_{1} \neq x_{2}<+1 .
\end{gathered}
$$

Consequently,

$$
\begin{aligned}
\left\langle\left(x^{2}-\tau_{i \zeta}^{2}\right)^{-1}\left(x^{2}-t_{i \zeta}^{2}\right)^{-1} \mathcal{U}, f\right\rangle= & \frac{f\left(t_{i \zeta}\right)+f\left(-t_{i \zeta}\right)}{t_{i \zeta}^{2}-\tau_{i \zeta}^{2}}-\frac{f\left(\tau_{i \zeta}\right)+f\left(-\tau_{i \zeta}\right)}{t_{i \zeta}^{2}-\tau_{i \zeta}^{2}} \\
& +P \frac{2}{\pi} \int_{-1}^{1} \frac{\sqrt{1-x^{2}}}{\left(x^{2}-t_{i \zeta}^{2}\right)\left(x^{2}-\tau_{i \zeta}^{2}\right)} f(x) d x .
\end{aligned}
$$

After some straightforward calculations, we obtain from (4.6) and (4.7)

$$
\begin{aligned}
\left\langle u^{-1}, f\right\rangle=\frac{1}{4} & \frac{\sqrt{1-4 \zeta^{2}}-1}{\sqrt{1-4 \zeta^{2}}}\left\langle\delta_{-t_{i \zeta}}+\delta_{t_{i \zeta}}, f\right\rangle+\frac{1}{4} \frac{\sqrt{1-4 \zeta^{2}}+1}{\sqrt{1-4 \zeta^{2}}}\left\langle\delta_{-\tau_{i \zeta}}+\delta_{\tau_{i \zeta}}, f\right\rangle \\
& +i \zeta P \frac{1}{\pi} \int_{-1}^{1} \frac{\sqrt{1-x^{2}}}{\left(t_{i \zeta}^{2}-x^{2}\right)\left(x^{2}-\tau_{i \zeta}^{2}\right)} f(x) d x
\end{aligned}
$$

From $\gamma_{1} u^{(1)}=-x^{2} u^{-1}$ and (4.8), we infer that

$$
\begin{aligned}
\left\langle u^{(1)}, f\right\rangle=\frac{1}{2} & \frac{i \zeta}{\sqrt{1-4 \zeta^{2}}}\left\langle\delta_{-t_{i \zeta}}+\delta_{t_{i \zeta}}, f\right\rangle-\frac{1}{2} \frac{i \zeta}{\sqrt{1-4 \zeta^{2}}}\left\langle\delta_{-\tau_{i \zeta}}+\delta_{\tau_{i \zeta}}, f\right\rangle \\
& +P \frac{1}{\pi} \int_{-1}^{1} \frac{x^{2} \sqrt{1-x^{2}}}{\left(t_{i \zeta}^{2}-x^{2}\right)\left(x^{2}-\tau_{i \zeta}^{2}\right)} f(x) d x
\end{aligned}
$$

(2) $\zeta=\frac{1}{2}$.

Then $t_{\frac{1}{2} i}^{2}=\frac{1}{2}, \tau_{\frac{1}{2} i}^{2}=\frac{1}{2}$. As above, we obtain 
or

$$
\begin{aligned}
u^{-1}= & \delta_{(\sqrt{2})^{-1}}-(\sqrt{2})^{-1}\left(x-(\sqrt{2})^{-1}\right)^{-1} \delta_{-(\sqrt{2})^{-1}}+\frac{1}{2}(i-1)\left(x^{2}-\frac{1}{2}\right)^{-1} \delta_{(\sqrt{2})^{-1}} \\
& -\frac{1}{2}(\sqrt{2})^{-1}(i-1)\left(x-(\sqrt{2})^{-1}\right)^{-1}\left(x^{2}-\frac{1}{2}\right)^{-1} \delta_{-(\sqrt{2})^{-1}}-\frac{1}{4} i\left(x^{2}-\frac{1}{2}\right)^{-2} \mathcal{U}
\end{aligned}
$$

$$
\begin{aligned}
u^{-1}= & \delta_{(\sqrt{2})^{-1}}-(\sqrt{2})^{-1}\left(x-(\sqrt{2})^{-1}\right)^{-1} \delta_{-(\sqrt{2})^{-1}}-\frac{1}{2}(i-1)\left(x+(\sqrt{2})^{-1}\right)^{-1} \delta_{(\sqrt{2})^{-1}}^{\prime} \\
& +\frac{1}{2}(\sqrt{2})^{-1}(i-1)\left(x-(\sqrt{2})^{-1}\right)^{-2} \delta_{-(\sqrt{2})^{-1}}^{\prime}-\frac{1}{4} i\left(x^{2}-\frac{1}{2}\right)^{-2} \mathcal{U} .
\end{aligned}
$$

On the other hand, we have

$$
\begin{aligned}
& \left\langle\left(x^{2}-\frac{1}{2}\right)^{-2} \mathcal{U}, f\right\rangle=\left\langle\mathcal{U}, \theta_{-(\sqrt{2})^{-1}}^{2} \theta_{(\sqrt{2})^{-1}}^{2} f\right\rangle
\end{aligned}
$$

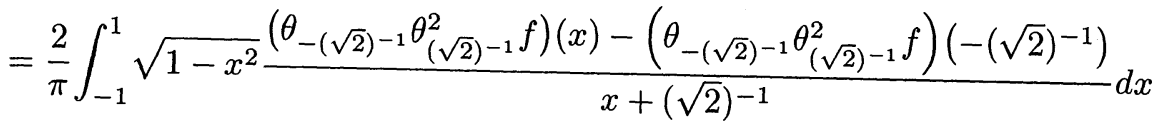

$$
\begin{aligned}
& =-\sqrt{2}\left(\theta_{-(\sqrt{2})^{-1}} \theta_{(\sqrt{2})^{-1}}^{2} f\right)\left(-(\sqrt{2})^{-1}\right) \\
& +P \frac{2}{\pi} \int_{-1}^{1} \frac{\sqrt{1-x^{2}}}{x+(\sqrt{2})^{-1}} \frac{\left(\theta_{-(\sqrt{2})^{-1}} \theta_{(\sqrt{2})^{-1}} f\right)(x)-\left(\theta_{-(\sqrt{2})^{-1}} \theta_{(\sqrt{2})^{-1}} f\right)\left((\sqrt{2})^{-1}\right)}{x-(\sqrt{2})^{-1}} d x \\
& =-\sqrt{2}\left(\theta_{-(\sqrt{2})^{-1}} \theta_{(\sqrt{2})^{-1}}^{2} f\right)\left(-(\sqrt{2})^{-1}\right)
\end{aligned}
$$

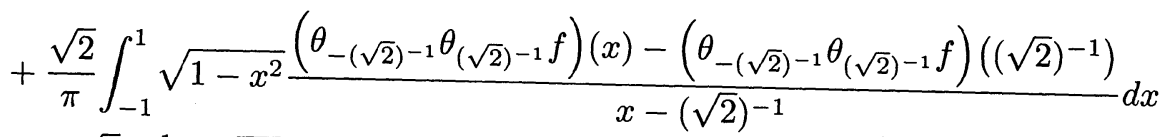

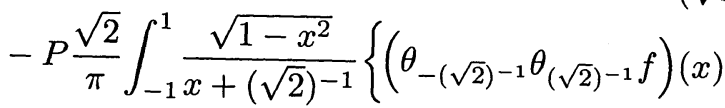

$$
\begin{aligned}
& \left.-\left(\theta_{-(\sqrt{2})^{-1}} \theta_{(\sqrt{2})^{-1}} f\right)\left((\sqrt{2})^{-1}\right)\right\} d x \\
& \left.=-\sqrt{2}\left(\theta_{-(\sqrt{2})^{-1}} \theta_{(\sqrt{2})^{-1}}^{2} f\right)\left(-(\sqrt{2})^{-1}\right)+2\left(\theta_{-(\sqrt{2})^{-1}} \theta_{(\sqrt{2})^{-1}} f\right)(\sqrt{2})^{-1}\right) \\
& +P \frac{\sqrt{2}}{\pi} \int_{-1}^{1} \frac{\sqrt{1-x^{2}}}{x-(\sqrt{2})^{-1}} \frac{\left(\theta_{(\sqrt{2})^{-1}} f\right)(x)-\left(\theta_{(\sqrt{2})^{-1}} f\right)\left(-(\sqrt{2})^{-1}\right)}{x+(\sqrt{2})^{-1}} d x \\
& -P \frac{\sqrt{2}}{\pi} \int_{-1}^{1} \frac{\sqrt{1-x^{2}}}{x+(\sqrt{2})^{-1}} \frac{\left(\theta_{-(\sqrt{2})^{-1}} f\right)(x)-\left(\theta_{-(\sqrt{2})^{-1}} f\right)\left((\sqrt{2})^{-1}\right)}{x-(\sqrt{2})^{-1}} d x \\
& =\frac{1}{\sqrt{2}}\left(f^{\prime}\left((\sqrt{2})^{-1}\right)-f^{\prime}\left(-(\sqrt{2})^{-1}\right)\right)+P \frac{\sqrt{2}}{\pi} \int_{-1}^{1} \frac{\sqrt{1-x^{2}}}{x^{2}-\frac{1}{2}} \frac{f(x)-f\left((\sqrt{2})^{-1}\right)}{x-(\sqrt{2})^{-1}} d x \\
& -P \frac{\sqrt{2}}{\pi} \int_{-1}^{1} \frac{\sqrt{1-x^{2}}}{x^{2}-\frac{1}{2}} \frac{f(x)-f\left(-(\sqrt{2})^{-1}\right)}{x+(\sqrt{2})^{-1}} d x
\end{aligned}
$$

on account of

$$
\frac{1}{\left(x+(\sqrt{2})^{-1}\right)\left(x-(\sqrt{2})^{-1}\right)}=\frac{\sqrt{2}}{2}\left\{\frac{1}{x-(\sqrt{2})^{-1}}-\frac{1}{x+(\sqrt{2})^{-1}}\right\} .
$$


But

$$
\frac{1}{\left(x+(\sqrt{2})^{-1}\right)^{2}\left(x-(\sqrt{2})^{-1}\right)}=-\frac{\sqrt{2} / 2}{\left(x+(\sqrt{2})^{-1}\right)^{2}}-\frac{1 / 2}{x+(\sqrt{2})^{-1}}+\frac{1 / 2}{x-(\sqrt{2})^{-1}} .
$$

Therefore,

$$
\begin{aligned}
\left\langle\left(x^{2}-\frac{1}{2}\right)^{-2} \mathcal{U}, f\right\rangle=\frac{1}{\sqrt{2}} & \left(f^{\prime}\left((\sqrt{2})^{-1}\right)-f^{\prime}\left(-(\sqrt{2})^{-1}\right)\right) \\
& +\frac{\sqrt{2}}{2}\left\{P \frac{\sqrt{2}}{\pi} \int_{-1}^{1} \frac{\sqrt{1-x^{2}}}{x-(\sqrt{2})^{-1}} \frac{f(x)-f\left((\sqrt{2})^{-1}\right)}{x-(\sqrt{2})^{-1}} d x\right. \\
& -\frac{1}{\pi} \int_{-1}^{1} \sqrt{1-x^{2}} \frac{f(x)-f\left((\sqrt{2})^{-1}\right)}{x-(\sqrt{2})^{-1}} d x \\
& \left.+P \frac{1}{\pi} \int_{-1}^{1} \frac{\sqrt{1-x^{2}}}{x+(\sqrt{2})^{-1}}\left(f(x)-f\left((\sqrt{2})^{-1}\right)\right) d x\right\} \\
& -\frac{\sqrt{2}}{2}\left\{-P \frac{\sqrt{2}}{\pi} \int_{-1}^{1} \frac{\sqrt{1-x^{2}}}{x+(\sqrt{2})^{-1}} \frac{f(x)-f\left(-(\sqrt{2})^{-1}\right)}{x+(\sqrt{2})^{-1}} d x\right. \\
& -\frac{1}{\pi} \int_{-1}^{1} \sqrt{1-x^{2}} \frac{f(x)-f\left(-(\sqrt{2})^{-1}\right)}{x+(\sqrt{2})^{-1}} d x \\
& \left.+P \frac{1}{\pi} \int_{-1}^{1} \frac{\sqrt{1-x^{2}}}{x-(\sqrt{2})^{-1}}\left(f(x)-f\left(-(\sqrt{2})^{-1}\right)\right) d x\right\}
\end{aligned}
$$

or

$$
\begin{aligned}
&\left\langle\left(x^{2}-\frac{1}{2}\right)^{-2} \mathcal{U}, f\right\rangle=\frac{1}{\sqrt{2}}\left(f^{\prime}\left((\sqrt{2})^{-1}\right)-f^{\prime}\left(-(\sqrt{2})^{-1}\right)\right) \\
&+\frac{\sqrt{2}}{2}\left\{P f \frac{\sqrt{2}}{\pi} \int_{-1}^{1} \frac{\sqrt{1-x^{2}}}{\left(x-(\sqrt{2})^{-1}\right)^{2}} f(x) d x\right. \\
&-f\left((\sqrt{2})^{-1}\right) P f \frac{\sqrt{2}}{\pi} \int_{-1}^{1} \frac{\sqrt{1-x^{2}}}{\left(x-(\sqrt{2})^{-1}\right)^{2}} d x \\
&-P \frac{1}{\pi} \int_{-1}^{1} \frac{\sqrt{1-x^{2}}}{x-(\sqrt{2})^{-1}} f(x) d x \\
&\left.\left.+P \frac{1}{\pi} \int_{-1}^{1} \frac{\sqrt{1-x^{2}}}{x+(\sqrt{2})^{-1}} f(x) d x-\frac{2}{\sqrt{2}} f(\sqrt{2})^{-1}\right)\right\} \\
&-\frac{\sqrt{2}}{2}\left\{-P f \frac{\sqrt{2}}{\pi} \int_{-1}^{1} \frac{\sqrt{1-x^{2}}}{\left(x+(\sqrt{2})^{-1}\right)^{2}} f(x) d x\right. \\
&+ f\left(-(\sqrt{2})^{-1}\right) P f \frac{\sqrt{2}}{\pi} \int_{-1}^{1} \frac{\sqrt{1-x^{2}}}{\left(x+(\sqrt{2})^{-1}\right)^{2}} d x \\
&-P \frac{1}{\pi} \int_{-1}^{1} \frac{\sqrt{1-x^{2}}}{x+(\sqrt{2})^{-1}} f(x) d x \\
&+\left.P \frac{1}{\pi} \int_{-1}^{1} \frac{\sqrt{1-x^{2}}}{x-(\sqrt{2})^{-1}} f(x) d x+\frac{2}{\sqrt{2}} f\left(-(\sqrt{2})^{-1}\right)\right\}
\end{aligned}
$$


It is a simple computation to see that

$$
\operatorname{Pf} \frac{\sqrt{2}}{\pi} \int_{-1}^{1} \frac{\sqrt{1-x^{2}}}{\left(x+(\sqrt{2})^{-1}\right)^{2}} d x=\operatorname{Pf} \frac{\sqrt{2}}{\pi} \int_{-1}^{1} \frac{\sqrt{1-x^{2}}}{\left(x-(\sqrt{2})^{-1}\right)^{2}} d x=-\sqrt{2} .
$$

Then,

$$
\begin{aligned}
\left\langle\left(x^{2}-\frac{1}{2}\right)^{-2} \mathcal{U}, f\right\rangle= & \frac{1}{\sqrt{2}}\left(f^{\prime}\left((\sqrt{2})^{-1}\right)-f^{\prime}\left(-(\sqrt{2})^{-1}\right)\right)-P \frac{2}{\pi} \int_{-1}^{1} \frac{\sqrt{1-x^{2}}}{x^{2}-\frac{1}{2}} f(x) d x \\
& +\operatorname{Pf} \frac{1}{\pi} \int_{-1}^{1} \frac{\sqrt{1-x^{2}}}{\left(x-(\sqrt{2})^{-1}\right)^{2}} f(x) d x \\
& +\operatorname{Pf} \frac{1}{\pi} \int_{-1}^{1} \frac{\sqrt{1-x^{2}}}{\left(x+(\sqrt{2})^{-1}\right)^{2}} f(x) d x
\end{aligned}
$$

With the results

$$
\begin{gathered}
\left\langle\delta_{(\sqrt{2})^{-1}}, f\right\rangle=f\left((\sqrt{2})^{-1}\right) \\
-\frac{1}{\sqrt{2}}\left\langle\left(x-(\sqrt{2})^{-1}\right)^{-1} \delta_{-(\sqrt{2})^{-1}}, f\right\rangle=\frac{1}{2}\left(f\left(-(\sqrt{2})^{-1}\right)-f\left((\sqrt{2})^{-1}\right)\right) \\
-\frac{1}{2}(i-1)\left\langle\left(x+(\sqrt{2})^{-1}\right)^{-1} \delta_{(\sqrt{2})^{-1}}^{\prime}, f\right\rangle=-\frac{1}{2}(i-1)\left\langle\delta_{(\sqrt{2})^{-1}}^{\prime}, \theta_{-(\sqrt{2})^{-1}} f\right\rangle \\
=\frac{1}{2}(i-1)\left\langle\delta_{(\sqrt{2})^{-1}}, D \theta_{-(\sqrt{2})^{-1}} f\right\rangle \\
=\frac{1}{2}(i-1)\left\langle\delta_{(\sqrt{2})^{-1}}, \theta_{-(\sqrt{2})^{-1}} f^{\prime}-\theta_{-(\sqrt{2})^{-1}}^{2} f\right\rangle \\
=\frac{1}{2}(i-1)\left\{\frac{\sqrt{2}}{2} f^{\prime}\left((\sqrt{2})^{-1}\right)-\frac{1}{2}\left(f\left((\sqrt{2})^{-1}\right)-f\left(-(\sqrt{2})^{-1}\right)\right)\right\}
\end{gathered}
$$

because $D \theta_{c}=\theta_{c} D-\theta_{c}^{2}$,

$$
\begin{aligned}
& \frac{\sqrt{2}}{4}(i-1)\left\langle\left(x-(\sqrt{2})^{-1}\right)^{-2} \delta_{-(\sqrt{2})^{-1}}^{\prime}, f\right\rangle=\frac{\sqrt{2}}{4}(i-1)\left\langle\delta_{-(\sqrt{2})^{-1}}^{\prime}, \theta_{(\sqrt{2})^{-1}}^{2} f\right\rangle \\
& =-\frac{\sqrt{2}}{4}(i-1)\left\langle\delta_{-(\sqrt{2})^{-1}}, D \theta_{(\sqrt{2})^{-1}}^{2} f\right\rangle \\
& =-\frac{\sqrt{2}}{4}(i-1)\left\langle\delta_{-(\sqrt{2})^{-1}},\left(\frac{1}{x-(\sqrt{2})^{-1}}\left\{\frac{f(x)-f\left((\sqrt{2})^{-1}\right)}{x-(\sqrt{2})^{-1}}-f^{\prime}\left((\sqrt{2})^{-1}\right)\right\}\right)^{\prime}\right\rangle \\
& =-\frac{\sqrt{2}}{8}(i-1)\left\{\sqrt{2}\left(f\left(-(\sqrt{2})^{-1}\right)-f\left((\sqrt{2})^{-1}\right)\right)+\left(f^{\prime}\left(-(\sqrt{2})^{-1}\right)+f^{\prime}\left((\sqrt{2})^{-1}\right)\right)\right\},
\end{aligned}
$$

we finally obtain from (4.10) and (4.12)

$$
\begin{aligned}
\left\langle u^{-1}, f\right\rangle=\frac{\sqrt{2}}{8}\left\langle\delta_{(\sqrt{2})^{-1}}^{\prime}-\delta_{-(\sqrt{2})^{-1}}^{\prime}, f\right\rangle+\frac{1}{2}\left\langle\delta_{(\sqrt{2})^{-1}}+\delta_{-(\sqrt{2})^{-1}}, f\right\rangle & \\
-\frac{i}{4} & \left\{\operatorname{Pf} \frac{1}{\pi} \int_{-1}^{1} \frac{\sqrt{1-x^{2}}}{\left(x+(\sqrt{2})^{-1}\right)^{2}} f(x) d x+P f \frac{1}{\pi} \int_{-1}^{1} \frac{\sqrt{1-x^{2}}}{\left(x-(\sqrt{2})^{-1}\right)^{2}} f(x) d x\right. \\
& \left.-P \frac{2}{\pi} \int_{-1}^{1} \frac{\sqrt{1-x^{2}}}{x^{2}-\frac{1}{2}} f(x) d x\right\} .
\end{aligned}
$$


From $\gamma_{1} u^{(1)}=-x^{2} u^{-1}$ where $\gamma_{1}=-\lambda=-\frac{1}{2} i$ and (4.13), we obtain

$$
\begin{aligned}
\left\langle u^{(1)}, f\right\rangle=-\frac{\sqrt{2}}{8} & i\left\langle\delta_{(\sqrt{2})^{-1}}^{\prime}-\delta_{-(\sqrt{2})^{-1}}^{\prime}, f\right\rangle+P \frac{1}{\pi} \int_{-1}^{1} x^{2} \frac{\sqrt{1-x^{2}}}{x^{2}-\frac{1}{2}} f(x) d x \\
- & \frac{1}{2}\left\{P f \frac{1}{\pi} \int_{-1}^{1} x^{2} \frac{\sqrt{1-x^{2}}}{\left(x+(\sqrt{2})^{-1}\right)^{2}} f(x) d x\right. \\
+ & \left.P f \frac{1}{\pi} \int_{-1}^{1} x^{2} \frac{\sqrt{1-x^{2}}}{\left(x-(\sqrt{2})^{-1}\right)^{2}} f(x) d x\right\} .
\end{aligned}
$$

\section{Representation of $u^{-1}, u^{(1)}, u^{(2)}$ in Example 3}

In the case of Example 3, we have

$$
S\left(u^{-1}\right)(z)=\frac{-1}{z+\frac{1}{2} S(v)(z)}:=L(z)
$$

where

$$
v=\mathcal{H} \quad \text { and } \quad S(v)(z)=\frac{1}{\sqrt{\pi}} \int_{-\infty}^{\infty} \frac{e^{-t^{2}}}{t-z} d t
$$

satisfies

$$
S^{\prime}(v)(z)=-2 z S(v)(z)-2 .
$$

As above, we have $\Im L(z)>0$ for $\Im z>0$ and

$$
L(z)=\int_{-\infty}^{\infty} \frac{d \mu(t)}{t-z}
$$

where $\mu$ is a non-decreasing function. This function is absolutely continuous with respect to Lebesgue measure since

$$
\Im L(z) \leq\left[y+\frac{1}{2 \sqrt{\pi}} \int_{-\infty}^{\infty} \frac{y e^{-t^{2}}}{(t-x)^{2}+y^{2}} d t\right]^{-1}
$$

But

$$
\begin{aligned}
\int_{-\infty}^{\infty} \frac{y e^{-t^{2}}}{(t-x)^{2}+y^{2}} d t & =\int_{-\infty}^{\infty} \frac{y e^{-(x+t)^{2}}}{t^{2}+y^{2}} d t \geq \int_{-1}^{1} \frac{y e^{-(x+t)^{2}}}{t^{2}+y^{2}} d t \\
& \geq 2 e^{-x^{2}-2|x|-1} \int_{0}^{1} \frac{y}{t^{2}+y^{2}} d t=2 e^{-(|x|+1)^{2}} \operatorname{Arctan} \frac{1}{y} \\
& \geq \frac{\pi}{2} e^{-(|x|+1)^{2}}
\end{aligned}
$$

for $0<y \leq 1$. Therefore,

$$
\Im L(z) \leq \frac{4}{\sqrt{\pi}} e^{(|x|+1)^{2}} \text { for } \quad 0<y \leq 1
$$

We infer for (5.2)

$$
L(z)=\frac{1}{2 \sqrt{\pi}} \int_{-\infty}^{\infty} \frac{e^{-t^{2}}}{\left(t-\int_{0}^{t} e^{-\left(t^{2}-\vartheta^{2}\right)} d \vartheta\right)^{2}+\left(\frac{\sqrt{\pi}}{2} e^{-t^{2}}\right)^{2}} \frac{d t}{t-z}, \quad y>0
$$

since

$$
P \frac{1}{2 \sqrt{\pi}} \int_{-\infty}^{\infty} \frac{e^{-t^{2}}}{t-x} d t=-\int_{0}^{x} e^{-\left(x^{2}-t^{2}\right)} d t, \quad x \in R
$$


Consequently,

$$
\left\langle u^{-1}, f\right\rangle=\frac{1}{2 \sqrt{\pi}} \int_{-\infty}^{\infty} \frac{e^{-x^{2}}}{\left(x-\int_{0}^{x} e^{-\left(x^{2}-t^{2}\right)} d t\right)^{2}+\frac{\pi}{4} e^{-2 x^{2}}} f(x) d x .
$$

We deduce that

$$
\left\langle u^{(1)}, f\right\rangle=\frac{1}{\sqrt{\pi}} \int_{-\infty}^{\infty} \frac{x^{2} e^{-x^{2}}}{\left(x-\int_{0}^{x} e^{-\left(x^{2}-t^{2}\right)} d t\right)^{2}+\frac{\pi}{4} e^{-2 x^{2}}} f(x) d x .
$$

The forms $u^{-1}$ and $u^{(1)}$ are really Laguerre-Hahn forms [6]. Indeed, from $S(v)(z)=$ $-2 z-\frac{2}{S\left(u^{-1}\right)(z)}$ and (5.1), we obtain

$$
S^{\prime}\left(u^{-1}\right)(z)=2 z^{2} S^{2}\left(u^{-1}\right)(z)+2 z S\left(u^{-1}\right)(z) .
$$

This Bernoulli equation shows that $u^{-1}$ is a Laguerre-Hahn form of class zero [3]. Further, from $\gamma_{1} S\left(u^{(1)}\right)(z)=-\frac{1}{S(u)(z)}-z$ and $z S^{\prime}(u)(z)=-2\left(z^{2}+1\right) S(u)(z)-2 z$, we get

$$
z S^{\prime}\left(u^{(1)}\right)(z)=z S^{2}\left(u^{(1)}\right)(z)-2\left(z^{2}-1\right) S\left(u^{(1)}\right)(z)-2 z .
$$

Here, the class is $s=1$ [1]. On the other hand, from (3.28), we have

$$
u^{(2)}=\frac{1}{2}\left(\delta+v^{(1)}\right) \text {. }
$$

As above, we easily obtain the following representation for $v^{(1)}$ [19], see also [2]

$$
\left\langle v^{(1)}, f\right\rangle=\frac{1}{2 \sqrt{\pi}} \int_{-\infty}^{\infty} \frac{e^{-x^{2}}}{\left(\int_{0}^{x} e^{-\left(x^{2}-t^{2}\right)} d t\right)^{2}+\frac{\pi}{4} e^{-2 x^{2}}} f(x) d x .
$$

The form $u^{(2)}$ is also a Laguerre-Hahn form of class $s=1$, since from (5.7)

$$
S\left(u^{(2)}\right)(z)=\frac{1}{2}\left\{-\frac{1}{z}+S\left(v^{(1)}\right)(z)\right\}=-\frac{1}{2 z}-\frac{1}{S(v)(z)}-z .
$$

Therefore, on account of (5.1), we obtain

$$
z S^{\prime}\left(u^{(2)}\right)(z)=-2 z S^{2}\left(u^{(2)}\right)(z)-2\left(z^{2}+1\right) S\left(u^{(2)}\right)(z)-2 z
$$

Acknowledgements. The authors wish to thank the referees for valuable comments and suggestions.

\section{References}

1. J. Alaya and P. Maroni, Description of symmetric Laguerre-Hahn forms of class $s=1$, to appear.

2. Richard Askey and Jet Wimp, Associated Laguerre and Hermite polynomials, Proc. Roy. Soc. Edinburgh 96A (1984), 15-37.

3. H. Bouakkaz and P. Maroni, Description des polynômes de Laguerre-Hahn de classe zero, Ann. Comp. Appl. Math. 9 (1991), 189-194.

4. T. S. Chihara, An Introduction to Orthogonal Polynomials, Gordon and Breach, New-York, 1978.

5. - Orthogonal polynomials and measures with end point masses, Rocky Mount. J. Math. 15 (1985), 705-719.

6. J. Dini and P. Maroni, La multiplication d'une forme linéaire par une fraction rationnelle. Application aux formes de Laguerre-Hahn, Ann. Polon. Math. 52 (1990), 175-185.

7. P. Duren, Theory of $H^{p}$ Spaces, Academic Press, New York, 1970.

8. F. Hamel, Inverse of co-recursive forms of Tchebychev forms, in preparation.

9. A. Magnus, Riccati acceleration of the Jacobi continued fractions and Laguerre-Hahn orthogonal polynomials, In: Pade Approximation and its Applications, Proc., Bad Honnef (1983), Lecture Notes in Math. (Eds. H. Werner and H.T. Bunger) Springer, Berlin (1984), 213-230. 
10. F. Marcellán and P. Maroni, Sur l'adjonction d'une masse de Dirac à une forme régulière et semi-classique, Ann. Mat. Pura ed Appl. 162 (1992), 1-22.

11. P. Maroni, Le calcul des formes linéaires et les polynômes orthogonaux semi-classiques, In: Orthogonal Polynomials and their Applications, M. Alfaro et. al., eds. Lecture Notes in Mathematics 1329, Springer, Berlin (1988), 279-290.

12. _ـ Sur la décomposition quadratique d'une suite de polynômes orthogonaux I, Rivista di Mat. pura ed appl. 6 (1990), 19-53.

13. - Une théorie algébrique des polynômes orthogonaux. Application aux polynômes orthogonaux semi-classiques, Ann. Comp. Appl. Math. 9 (1991), 95-130.

14. - Variations around classical orthogonal polynomials. Connected problems, J. Comp. Appl. Math. 48 (1993), 133-155.

15. _ An introduction to second degree forms, Adv. Comp. Math. 3 (1995), 59-88.

16. $\ldots$ On a regular form defined by a pseudo-function, Numer. Algorithms 11 (1996), 243-254.

17. F. Peherstorfer, On Bernstein-Szegö orthogonal polynomials on several intervals, SIAM J. Math. Anal. 21 (1990), 461-482.

18. G. Sansigre and G. Valent, A large family of semi-classical polynomials: the perturbed Tchebychev, J. Comp. Appl. Math. 57 (1995), 271-281.

19. J. A. Shohat and J. D. Tamarkin, The problem of moments, Math. Surveys 1, Amer. Math. Soc., Providence, New York, 1950.

Ecole Nationale d'Ingenieurs de Gabes, Route de Medenine, 6029-Gabes, Tunisie

Universite Pierre et Marie Curie-C.N.R.S., Laboratoire D'Analyse Numerique, 4 Place Jussieu, 75252 Paris Cedex 05, France 